\title{
On Pre-connected sets in Bitopological Spaces
}

\author{
Alaa Erees \\ Dept. of Mathematics - College of science - Kufa University
}

\begin{abstract}
In this paper,we give and study a generalization for the concept "pre-connected set" in bitopological spaces, through as well as we give several various results related to this generalization .
\end{abstract}

\section{Introduction}

The study of pre-connected sets in Bitopological Space takes place at several sources and researchers like Kelly (1963) , Valiru (1977) , Jelles (1989) , Al-Swidi (1993) and Dontcher (1998) .A generalization of the definition of the pre-connected set is presented with some theorems and examples to explain and illustrative it .Conclusions are added at the end of it.

\section{Some definitions}

Definition (1) :[ 2 ]

Let $(X, \tau)$ be a topological space. A subset $A$ of $X$ is said to be pre-open set iff $A \subseteq \operatorname{int}_{\tau}\left(c l_{\tau}(A)\right)$. The family of all pre-open sets is denoted by $p r-O(X)$.

Definition (2) : [ 3 ]

Let $(X, \tau, \rho)$ be a bitopological space. A subset $A$ of $X$ is called pre-open set with respect to the two topologies $\tau, \rho$ if $A \subseteq \operatorname{int}_{\tau}\left\lfloor l_{\rho}(A)\right\rfloor$.
The collection of all pre-open sets with respect to the two topologies $\tau$ and $\rho$ is denoted by $p r-O(X)$.

Definition (3) :[ 2 ]

Let $(X, \tau, \rho)$ be a bitopological space . A subset $A$ of $X$ is called pre-closed set of $X$ iff the complement of $A$ is pre-open set of $X$. The collection of all $p r$-closed sets with respect to the two topologies $\tau$ and $\rho$ denoted by $p r-C(X)$.

Note (1) :

The family of all $p r$-open sets of $X$ is not necessary a topology on $X$, see the following example .

Example (1) :

Let $X=\{a, b, c\}, \tau=\{X, \phi,\{a\}\}, \quad$ and $\rho=\{X, \phi,\{c\}\} .(X, \tau), \quad(X, \rho)$ are two topological spaces.Then $(X, \tau, \rho)$ is bitopological space, so :

$$
p r-O(X)=\{X, \phi,\{a\},\{b\},\{a, b\},\{a, c\},\{b, c\}\},
$$


Definition (4) :[ 6 ]

Let $(X, \tau, \rho)$ be a bitopological space.Two non- empty subsets $A$ and $B$ of $X$ are said to be $p r$-separated iff $[A \cap p r-c l(B)] \cup[B \cap p r-c l(A)]=\phi$ That is $A$ and $B$ are $p r-$ separated sets iff $A$ and $B$ are disjoint and every one contains no limit points of the other with respected to $p r-O(X)$.

Example (2) :

Let $X=\{a, b, c\}, \tau=\{X, \phi,\{a\},\{a, b\}\}$ and $\rho=\{X, \phi,\{c\},\{a, b\}\} .(X, \tau),(X, \rho)$ are two topological spaces, then $(X, \tau, \rho)$ is a bitopological space, so, $p r-O(X)=\{X, \phi,\{a\},\{b\},\{a, b\},\{a, c\},\{b, c\}\}$, Let $A=\{a\}$ and $B=\{b\}$ are two $p r$-open sets.

Hence $A$ and $B$ are $p r$-separated sets, since

$p r-c l(A)=p r-c l(\{a\})=\{a\}, p r-c l(B)=p r-(\{b\})=\{b\}$, and $[A \cap p r-c l(B)] \cup[p r-c l(A) \cap B]=$ $[\{a\} \cap\{b\}] \cup[\{a\} \cap\{b\}]=\phi \cup \phi=\phi$.

So, $A$ and $B$ are $p r$-separated subsets of $X$

Definition (5) :[ 6 ]

Let $(X, \tau, \rho)$ be a bitopological space. $\mathbf{A}$ subset $A$ of $X$ is said to be $p r$-disconnected set iff it is the union of two $p r$-separated non-empty sets in $X$, that is $\exists$ two non-empty sets $C$ and $D$ in $X$ such that $p r-c l(C) \cap D=\phi$

$C \cap p r-c l(D)=\phi$ and $A=C \bigcup D$.

$A$ is called $p r$-connected iff it is not $p r$-disconnected set .

Example (3) :

Let

$X=\{a, b, c\}, \tau=\{X, \phi,\{a\},\{b\},\{a, b\}\}$ and
$\rho=\{X, \phi,\{b\},\{a, c\}\} .(X, \tau),(X, \rho)$ are two topological spaces. Then $(X, \tau, \rho)$ is a bitopological space, so, $p r-O(X)=\{X, \phi,\{a\},\{b\},\{a, b\},\{b, c\}\}$,

Let $A=(a, b)$ and $C=(a), D=(b)$ so $A=C U D$.

Hence $p r-d(C)=a|, p r-d(D)=| b \mid$,

$[p r-d](C) \cap D] \cup[C \cap p r-d(D)]=[i a) \cap(b)] \cup[i a \cap(b]=\phi$.

Therefore $A$ is pr - plisconnected set, but if $B=\langle b, c| C=\{b, D=\langle b, c|$

hence $p r-d(C)=(b), p r-c)(D)=(b, c)$

$[p r-d(C, \cap D] \cup[C \cap p r-d(D)]=[(b) \cap(b, c)] \cup[b) \cap b, c]=\{b) \cup(b)=(b) \neq \phi$

Then $B$ is $p r$-connected set.

Notes (2) :

1. The empty set is trivially $p r$-connected .

2. Every singleton $p r-$ open set is $p r$-connected set, since it can not be expressed as a union of two non-empty $p r$-separated sets .

Definition (6) :[ 6 ]

Two points $a, b$ of a bitopological space $(X, \tau, \rho)$ are said to be $p r$-connected iff they are contained in a $p r-$ connected subset of $X$.

Definition (7) :[ 4 ]

Let $(X, \tau, \rho)$ be a bitopological space, and $Y$ be a subset of $X$. The relative bitopological space for $Y$ is denoted by $\left(Y, \tau_{Y}, \rho_{Y}\right)$, such that :

$\tau_{Y}=\{G \bigcap Y: G \in \tau\}$

$\rho_{Y}=\{H \cap Y: H \in \rho\}$

$\left(Y, \tau_{Y}, \rho_{Y}\right)$ is called a subspace of the bitopological space $(X, \tau, \rho)$, the relative bitopological space for $Y$ with respect to $p r$-open sets is the collection $p r-O(X)_{Y} \quad$ given by $p r-O(X)_{Y}=\{G \cap Y: G \in p r-O(X)\}$ 
2. Various results :

Theorem (1) :

Let $\left(Y, \tau_{Y}, \rho_{Y}\right)$ be subspace of $(X, \tau, \rho)$ let $A, B \subset p r-O(X)$ then $A$ and $B$ are $p r-O(X)$-separated iff they $p r-O(X)_{Y}$-separated.

Theorem (2) :

Let $\left(Y, \tau_{Y}, \rho_{Y}\right)$ be a subspace of bitopological $(X, \tau, \rho)$ and $A \subset Y$.Then $A$ is $p r$-disconnected iff it is $p r_{Y}$ disconnected .

Proof :

By above theorem, therefore, $A$ is the union of two $p r$-separated sets iff it is the union of two $p r_{\mathrm{Y}}$-separated sets .

Theorem (3) :

A bitopological space $(X, \tau, \rho)$ is $p r$-disconnected iff $\exists$ a non-empty proper subset of $X$ which is both $p r$ open and $p r-$ closed in $X$.

Proof :

Let $A$ a non-empty proper subset of $X$ which both $p r$-open and $p r$-closed.We will show that $X$ is a $p r$-disconnected .

Let $B=A^{c} \Rightarrow B \neq \phi$, since $A \subset X$, so $B \bigcup A=X$ and $A \cap B=\phi \ldots . . .(*)$

Since $A$ is both $p r$-open and $p r-$ closed $\Rightarrow B$ is both $p r$-open and $p r$-closed , hence $p r-c l(A)=A, p r-c l(B)=B$.

Thus $X$ is $p r$-disconnected.
Let $X$ is $p r$-disconnected ,Then $\exists$ two non-empty $p r$-separated subsets $A$ and $B \ni X=A \cup B$ ..(**)

$p r-c l(A) \cap B=\phi$ and $A \cap \operatorname{pr}-\operatorname{cl}(B)=\phi$, since $\quad A \subset \operatorname{pr}-\operatorname{cl}(A) \quad$ and $p r-c l(A) \cap B=\phi \Rightarrow A \cap B=\phi$.

Hence , $A=B^{c}$, by (**) , $B \neq \phi \Rightarrow X \subseteq A \cup p r-c l(B) \quad$ since $(B \subset \operatorname{pr}-\operatorname{cl}(B))$.

But

$A \bigcup p r-c l(B) \subseteq X \Rightarrow X=A \cup p r-c l(B)$ , also

$A \cap p r-c l(B)=\phi \Rightarrow A=[p r-c l(B)]^{c}$.

Similarity , $B=[p r-c l(A)]^{c}$. Since $p r-c l(A)$ and $\quad p r-c l(B)$ are $p r$-closed sets, therefore $A$ and $B$ are $p r$-open sets, and hence $A=B^{c}$ is a $p r$-closed set (since $A \cap B=\phi$ ). Thus $A$ is a non-empty $p r$-closed and $p r$-open set .

\section{Theorem (4) :}

Let $(X, \tau, \rho)$ be a bitopological space.Then $X$ is a $p r$-disconnected iff any one of the following statements holds :

i. $\quad X$ is the union of two non-empty disjoint $p r-$ open sets .

ii. $\quad X$ is the union of two non-empty disjoint $p r-$ closed sets .

\section{Proof :}

Let $X$ be a $p r$-disconnected $\Rightarrow \exists A \neq \phi, A \subset X$ which is both $p r$-closed and $p r-$ open set .Therefore $A^{c}$ is also both $p r-c l o s e d$ and $p r-$ open set and $A \cup A^{c}=X$. 
Hence the sets $A$ and $A^{c}$ are satisfy (i) and (ii) .

Let $X=A \cup B$ and $A \cap B=\phi$, where $A$ and $B$ are non-empty $p r$-open $\left(p r\right.$-closed) sets $\Rightarrow A=B^{c}$,so that $A$ is $p r$-closed( $p r-$ open)set.

Since

$B \neq \phi, B \subset X \Rightarrow A \neq \phi, A \subset X$ which is both $p r$-open and $p r$-closed .

Hence $X$ is a $p r$-disconnected.

Theorem (5) :

Let $E$ be a $p r$-connected subset of a bitopological space $(X, \tau, \rho)$.If $F \subset X$ such that $E \subset F \subset p r-c l(E)$, then $F$ is a $p r$-connected . In particular, $p r-c l(E)$ is $p r-$ connected .

Proof :

Suppose that $F$ is $p r$-disconnected $\Rightarrow A A, B \neq \phi, A \mid p r-d(B)=\phi, P P-d(A) \cap B=\phi, A \cup B=7$, since $\mathbb{B} \subset \mathbb{P}=A \| B \Rightarrow \mathbb{E} \subset A$ or $\mathbb{Z \subset D}$.

Let $\delta \subset A \Rightarrow p r-d(E) \subset p r-d(A)=p r-d(D) \cap B \subset p-d(A) \cap B=\phi \Rightarrow$ $p r-d(D) \cap B=$

Also, $A \cup B=P \subset p P-C(D)=B \subset \bar{P} \subset p P-d(D)=$ $p r-d(B) \cap B=B$

By (*) and (**) we get $B=\phi$ which is a contradiction, since $B \neq \phi$.

Hence $F$ is a $p r$-connected . Again, since $E \subset p r-c l(E) \subseteq p r-c l(E) \Rightarrow$ $p r-c l(E)$ is a $p r-$ connected .

Theorem (6) :

Let $(X, \tau, \rho)$ be a bitopological space ,and $E$ is a subset of $X$.If each two points of $E$ are $p r$-connected in some $p r$-connected subset of $E$, then $E$ is a $p r$-connected subset of $X$.

Proof :

Suppose that $E$ is not $p r-$ connected, then $\exists A, B \neq \phi \quad$ and $A, B \subset X \ni A \cap p r-c l(B)=\phi \quad, \quad$ and $p r-\operatorname{cl}(A) \cap B=\phi, E=A \cup B \quad, \quad$ since $A, B \neq \phi \Rightarrow \exists a \in A$ and $b \in B$ such that $a$ and $b$ must be contained in some $p r$-connected subset $F$ of $E$ since $F \subset A \cup B \Rightarrow$ either $F \subset A$ or $F \subset B \Rightarrow$

either $a, b \in A$ or $a, b \in B$. Let $a, b \in A$, since $b \in B \Rightarrow A \cap B \neq \phi$, which is a contradiction, since $A$ and $B$ are disjoint - Hence $E$ must be a $p r$-connected .

Theorem (7) :

Let $\left\{G_{\lambda}: \lambda \in \wedge\right\}$ be a family of $p r$-connected subsets of a bitopological space $(X, \tau, \rho)$ such that $\cap\left\{G_{\lambda}: \lambda \in \wedge\right\} \neq \phi$. Then $\bigcup\left\{G_{\lambda}: \lambda \in \wedge\right\}$ is a $p r-$ connected set .

Proof :

Suppose that $E=\bigcup\left\{G_{\lambda}: \lambda \in \wedge\right\}$ is a $p r$-disconnected $\Rightarrow \exists$ two non-empty disjoint sets $G_{1}$ and $G_{2}$ both $p r-o p e n$ in the subset $E$ of $X \ni E=G_{1} \cup G_{2}$, $\forall \lambda, G_{1} \cap G_{\lambda}, G_{2} \cap G_{\lambda}$ are disjoint sets both $p r-$ open in the subset $G \lambda$ э $\left(G_{1} \cap G_{\lambda}\right) \cup\left(G_{2} \cap G_{\lambda}\right)=\left(G_{1} \cup G_{2}\right) \cap G_{\lambda}=G_{\lambda}$ (*)

Since $G_{\lambda}$ is a $p r$-connected, one of the sets $G_{1} \cap G_{\lambda}$ and $G_{2} \cap G_{\lambda}$ must be empty , say $G_{1} \cap G_{\lambda}=\phi$, then by (*) $G_{2} \cap G_{\lambda}=G_{\lambda} \Rightarrow G_{\lambda} \subset G_{2}$. 
$\forall \lambda \in \wedge \Rightarrow \bigcup\left\{G_{\lambda}: \lambda \in \wedge\right\} \subset G_{2} \Rightarrow G_{1} \cup G_{2} \subset G_{2} \Rightarrow G_{1}=\phi$

, which is contradiction .

Hence $E$ must be $p r$-connected since

$G_{1}$ is non-empty , so $E$ is $p r$-connected .

Theorem (8) :

Let $\left\{G_{\lambda}: \lambda \in \wedge\right\}$ be a family of $p r$-connected subsets of a

bitopological space $(X, \tau, \rho)$ such that one of the member of this family intersects every other member, then $\bigcup G_{\lambda}$ is a $p r$-connected set . $\lambda \in \wedge$

Proof :

Let $G_{\lambda_{0}}$ be a fixed member of the given family $\ni G_{\lambda \circ} \cap G_{\lambda} \neq \phi, \forall \lambda \in \wedge \quad$ then $D_{\lambda}=G_{\lambda_{0}} \cup G_{\lambda}$ is a $p r-$ connected set $\forall \lambda \in \wedge$ by previous theorem .

Now

$\bigcup\left\{D_{\lambda}: \lambda \in \wedge\right\}=\bigcup\left\{G_{\lambda \circ} \cup G_{\lambda}: \lambda \in \wedge\right\}=$ $G_{\lambda_{0}} \bigcup\left[\bigcup\left\{G_{\lambda}: \lambda \in \wedge\right\}\right]=\bigcup\left\{G_{\lambda}: \lambda \in \wedge\right\}$

and

$\bigcap\left\{D_{\lambda}: \lambda \in \wedge\right\}=\bigcap\left\{G_{\lambda \circ} \cup G_{\lambda}: \lambda \in \wedge\right\}=$ $\bigcap\left\{D_{\lambda}: \lambda \in \wedge\right\}=G_{\lambda_{0}} \cup\left[\cap\left\{G_{\lambda}: \lambda \in \wedge\right\}\right] \neq \phi$ (always), since by our assumption $G_{\lambda_{0}} \neq \phi$ is intersects every $G_{\lambda} \neq \phi$ as $G_{\lambda_{0}} \cap G_{\lambda} \neq \phi, \forall \lambda \in \wedge$.

Hence by previous theorem $\bigcup\left\{D_{\lambda}: \lambda \in \wedge\right\}=\bigcup\left\{G_{\lambda}: \lambda \in \wedge\right\} \quad$ is $\quad$ a $p r-$ connected set .

\section{References :}

[ 1 ] Al-Swidi,Luay,and Shaker, A. Y. "Semi compactness in bitopological spaces" , to appear in the magazine of technical institutes establishment (1993).

[ 2 ] Dontcher, J. "Survey On Preopen sets", ar 14, Math 30 (1998), 1-18.

[ 3 ] Kelly, J. C. "Bitopological spaces", proc. London Math.Soc 13(1963), 71-89.

[ 4 ] Kelly, J. C. " Bitopological space", publ Math Debrecen,15,1968,87-90.

[ 5 ]Njastad , 0.,"ON some classes of nearly open sets" ,pacific, J. Math. 15(1965), 961970. [ 6 ] Valeriu , P., "On Some Properties of Bitopological Semi Separation space", (1977) ,71-77.

[ 7 ] Tapi , U.D. and Thakur, S.S and Sonwalkar, "Quasi-preopen sets" J. Indian Acad. Math ., 17 (1) (1995), 8-12 .
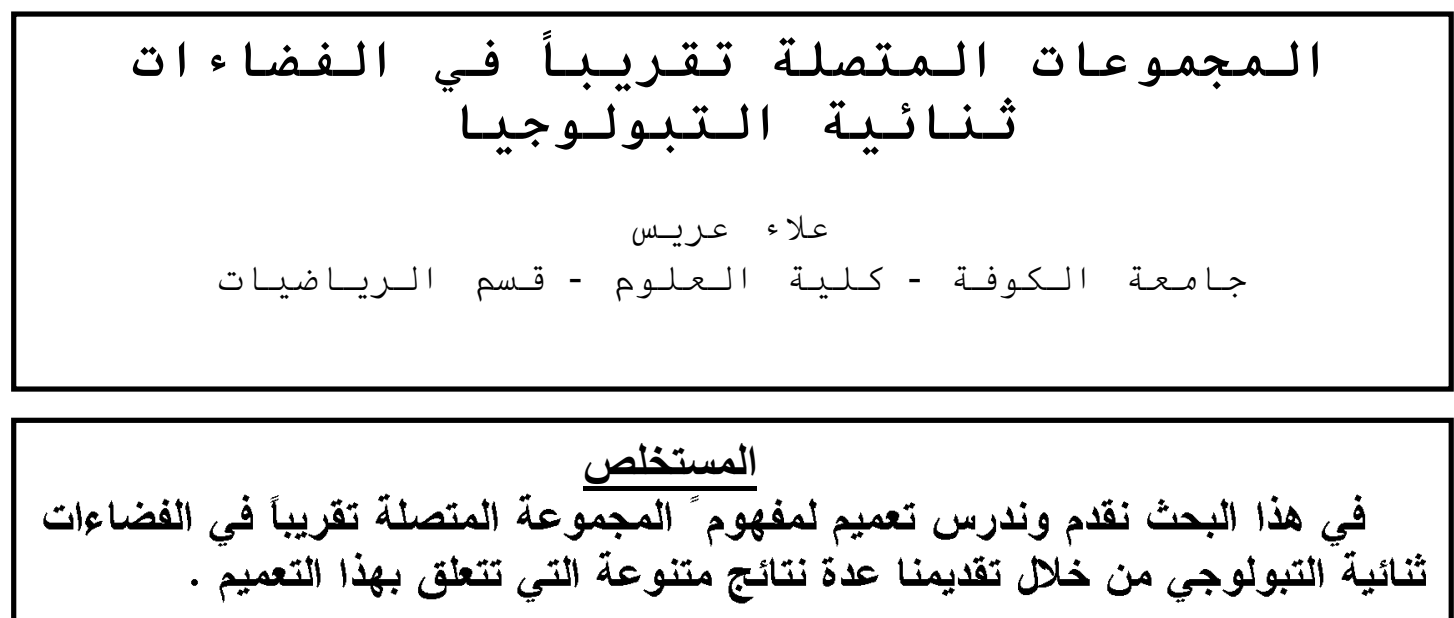